\title{
Diagnostic parameters of modified two-tier testing in European patients with early Lyme disease
}

\author{
ME Baarsma $^{1}$ (D) JFP Schellekens ${ }^{2,3} \cdot$ BC Meijer $^{2} \cdot$ AH Brandenburg $^{4} \cdot$ T. Souilljee $^{2} \cdot$ A Hofhuis $^{5} \cdot$ JW Hovius $^{1}$. \\ AP van Dam ${ }^{6,7}$
}

Received: 4 March 2020 / Accepted: 9 June 2020 / Published online: 6 July 2020

(C) The Author(s) 2020

\begin{abstract}
Modified two-tier testing (MTTT) for Lyme borreliosis (i.e. confirmation with an EIA instead of an immunoblot) has been shown to have improved sensitivity compared with standard two-tier testing (STTT) in samples from American patients, without losing specificity. The current study assesses the sensitivity and specificity of various algorithms of MTTT in European patients with erythema migrans $(E M)$ as a model disease for early Lyme borreliosis, and in appropriate controls. Four different immunoassays were used in the first tier, followed by either an immunoblot or the C6-EIA, or were used as standalone single-tier test. These tests were performed on consecutively collected sera of 228 Dutch patients with physician-diagnosed EM in the setting of general practice, 231 controls from the general population, and 50 controls with potentially cross-reactive antibodies. All the variants of MTTT that were studied had significantly higher sensitivity compared with their equivalent STTT, while retaining comparable specificity. Within the MTTT algorithms, classifying equivocal results as positive yielded better diagnostic parameters than classifying equivocal results as negative. The best diagnostic parameters were found using the Enzygnost-2 assay in the first tier, followed by a C6-ELISA in the second tier (sensitivity 77.6\%, 95\% CI 71.7-82.9; specificity 96.1\%, 95\% CI 92.7-98.2). This algorithm performed significantly better than the equivalent STTT algorithm in terms of sensitivity $(p<0.001)$, while maintaining comparable specificity (population controls $p=0.617$ ). Our results show that MTTT can be a useful tool for the serodiagnosis of European patients with early Lyme borreliosis.
\end{abstract}

Keywords Lyme disease $\cdot$ Borreliosis $\cdot$ Serology $\cdot$ Modified two-tier testing $\cdot$ C6

\section{Introduction}

While a thorough clinical assessment is a physician's foremost tool for diagnosing Lyme disease (Lyme borreliosis, LB), laboratory work-up is oftentimes required. The cornerstone of

Electronic supplementary material The online version of this article (https://doi.org/10.1007/s10096-020-03946-0) contains supplementary material, which is available to authorized users.

ME Baarsma

lyme@amc.nl

1 Amsterdam UMC, University of Amsterdam, Center for Experimental and Molecular Medicine, Amsterdam Infection \& Immunity, Meibergdreef 9, Amsterdam $1105 \mathrm{AZ}$, the Netherlands

2 Certe Laboratory of Infectious Diseases, Groningen, the Netherlands

3 Centre for Infectious Diseases Research, Diagnostics and Laboratory Surveillance, National Institute of Public Health and the Environment (RIVM), Bilthoven, the Netherlands this laboratory work-up is serology. Traditionally, guidelines in both the USA and Europe have advised to perform serodiagnosis of LB by applying two-tier testing [1-3]. In the first tier, a highly sensitive (but possibly false-positive) enzyme immunoassay (EIA) is performed, after which

4 Izore, Centrum Infectieziekten Friesland, Leeuwarden, the Netherlands

5 Epidemiology and Surveillance Unit, Centre for Infectious Disease Control, National Institute of Public Health and the Environment (RIVM), Bilthoven, the Netherlands

6 Department of Medical Microbiology, OLVG, Amsterdam, the Netherlands

7 Amsterdam UMC, University of Amsterdam, Department of Medical Microbiology, Amsterdam, Netherlands 
positive or equivocal results require confirmation in a second tier by $\operatorname{IgM} / \operatorname{IgG}$ immunoblotting. While some have come close, no single test has to the best of our knowledge attained or surpassed the combined sensitivity and specificity of standard two-tiered testing (STTT).

An important component of several EIAs is the VlsE protein. VlsE is highly immunogenic and antibodies are produced early after onset of infection with Borrelia burgdorferi sensu lato (Bbsl) $[4,5]$. Within the VlsE protein, a 26-amino acid sequence named invariable region 6 (IR6) has been shown to be highly conserved among the various subspecies of Bbsl, and to be highly immunogenic [6]. This had led to development of the C6-ELISA in which a synthetic peptide based on IR6 is used as antigen. The C6-ELISA is used both in the USA and Europe [7, 8].

For the second tier, American healthcare providers rely on immunoblots prepared from native cultivated B. burgdorferi sensu stricto (Bbss) bacteria, which is the primary causative agent in North America. In Europe, LB is caused by a variety of Bbsl species (e.g., B. garinii (Bgar) or B. afzelii (Bafz)) [9]. It is, therefore, virtually impossible to standardize immunoblotting in Europe using Borrelia lysates with respect to choice of antigen and uniform interpretation criteria. As a result, European immunoblots rely primarily on recombinant antigens of the various subspecies of Bbsl prevalent on that continent [10]. Other drawbacks of immunoblotting are that it is considered laborious and may be prone to inter-assay variation as it is non-quantitative.

A further argument for revisiting the STTT algorithm is its limited sensitivity early in the course of the disease, specifically for diagnosing early localized LB (an erythema migrans, EM). A meta-analysis found that STTT has a sensitivity of approximately 50\% in patients with an EM [11]. For this reason, most guidelines consider EM a clinical diagnosis and recommend against performing serological testing for early localized LB [1-3], even though extra certainty in the form of laboratory testing can be desirable, for instance when cutaneous lesions are atypical or vague.

Various solutions to the aforementioned problems with confirmatory testing by immunoblot have been proposed. In an American study comparing the diagnostic parameters of STTT vs. the C6-ELISA alone, the C6 assay was shown to be significantly more sensitive than standard two-tiered testing in EM, and comparably sensitive in various forms of disseminated LB [12]. However, this did come at the cost of a significantly lower specificity, even though the difference was small (C6 98.8\%; STTT 99.5\%) [12]. Other studies have examined the diagnostic parameters of so-called modified twotier testing (MTTT) using different EIAs or ELISAs in both tiers [7, 13-15]. These studies showed that the MTTT algorithm had far better sensitivity in early LB than STTT, but without the associated loss of specificity. These findingsand others - have led the FDA to recently approve a
Borrelia-EIA for use as confirmatory 2nd-tier test [16]. Previous research on MTTT has, however, been limited to sera from the USA. The differences in genospecies and diagnostic tests between Europe and the USA necessitate that these findings be reproduced in European sera, before MTTT algorithms can also be used on that continent.

In this multiple-gate case-control study, we have investigated the sensitivity of various assays and algorithms of MTTT in sera of European (Dutch) patients with physiciandiagnosed EM as the most prevalent manifestation of early LB in Europe, and their specificity in a variety of control sera. We have used one of several commercial EIAs in the first tier, followed by a European immunoblot or the C6-ELISA in the second tier. Similar to findings from the USA, we hypothesize that MTTT will improve sensitivity of serological testing in early LB over STTT, while maintaining adequate specificity.

\section{Materials and methods}

\section{EM sera}

Sera from 228 patients with early localized LB (an EM) were used as cases. These were selected from all sera sent between October 2010 and October 2011 by general practitioners (GPs) requesting Borrelia serology at the Certe Laboratory of Infectious Diseases (Certe LID) in Groningen, the Netherlands. Clinical data were collected from GPs by questionnaire, which were sent to the GP before serological test results were available. Selection of participants was consecutive: the study was performed on sera from all patients (1) who had sera sent in during the given timeframe, (2) for whom the necessary clinical information was available, (3) who had a probable EM $>5 \mathrm{~cm}$ as diagnosis, and (4) for whom enough serum was available to perform all tests. All other sera were excluded. The EM diagnosis was made by the GP, based on Dutch guidelines [17]. It was made purely based on these clinical findings, and hence independently from serological test results. Sera were drawn at the time of the clinical diagnosis. The process of serum selection is also given in Fig. 1. Of the selected EM sera, $46.1 \%$ were collected from males and $53.9 \%$ from females. The median age of patients was 53 years (range 1 to 86 , IQR 39-62 years). The aforementioned sample size was chosen independent from a sample size calculation.

\section{Population control sera}

Two hundred thirty-one sera were selected from the serum bank archived at the National Institute of Public Health and the Environment of the Netherlands (RIVM) and were used as population controls (PopC) (Fig. 1) [18]. These sera were 
Fig. 1 Serum selection

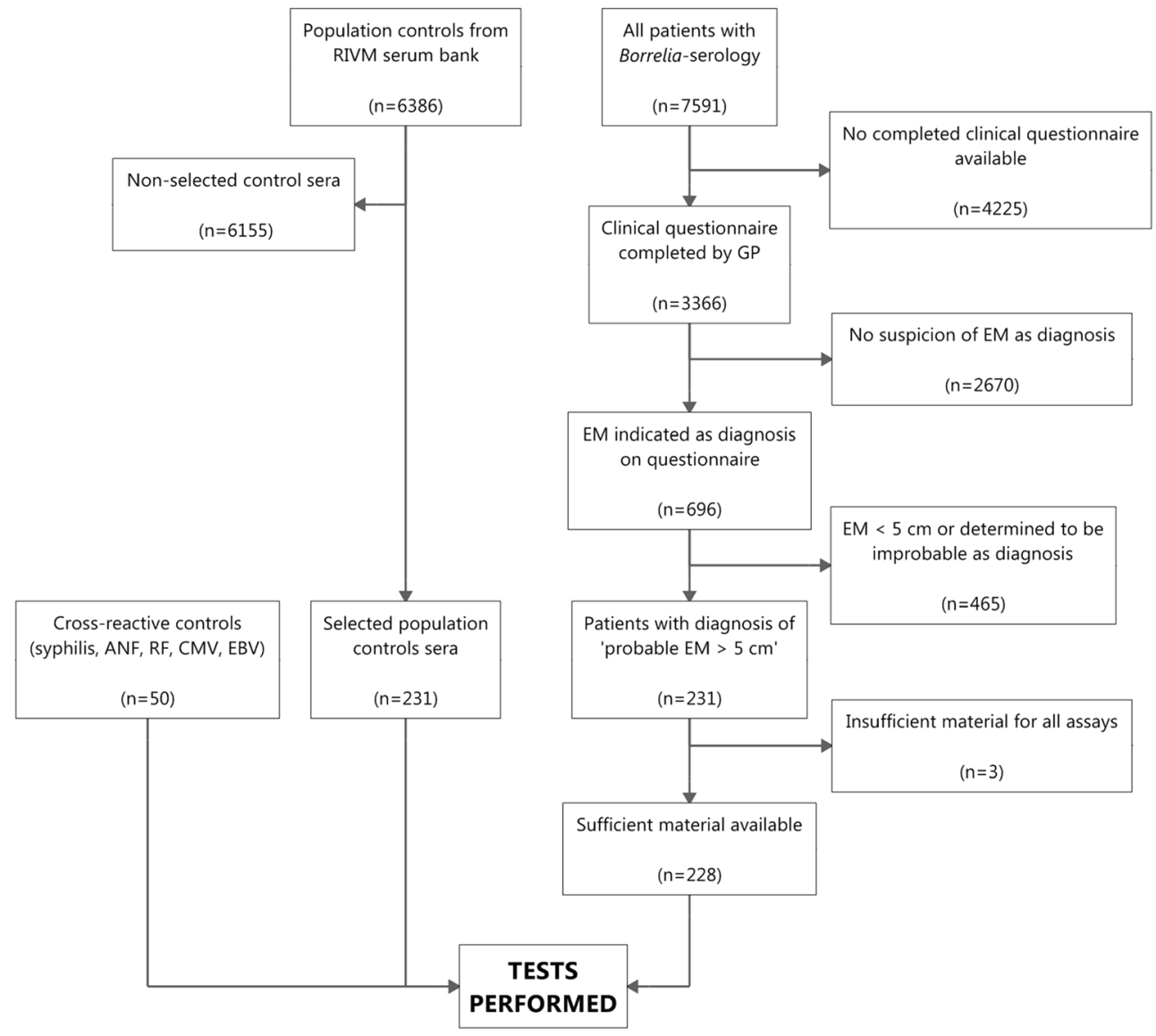

collected between 2006 and 2007 as a representative sample of the general population of the Netherlands. PopC sera were selected to yield an age and sex distribution comparable with the EM patients. Of the selected control sera, $45.9 \%$ were collected from males and $54.1 \%$ from females. The median age of population controls was 53 years (range 1 to $79, \mathrm{IQR}$ 40-62 years).

\section{Cross-reactive sera}

Ten sera with VDRL $\geq 1: 32$ and positive TPPA (syphilis), 10 sera with positive anti-nuclear factor (ANF), 10 sera with positive rheumatoid factor (RF), 10 sera positive for $\operatorname{IgM}$ and $\mathrm{IgG}$ antibodies to $\mathrm{CMV}$, and 10 sera positive for $\mathrm{IgM}$ and $\mathrm{IgG}$ antibodies to EBV were used as cross-reactive controls (CRC) (Fig. 1). These sera were selected from the serum bank of Certe LID.

\section{Assays}

Four commercially available immunoassays were performed on all samples (Table 1) in a non-blinded fashion. All sera which gave an equivocal or positive result in at least one of the EIAs/CLIA were tested by immunoblot. All assays were performed according to the manufacturers' instructions. The
Enzygnost-2 and immunoblot on EM sera were performed as part of normal clinical routine. All other assays were performed in batches, as were the Enzygnost-2 and immunoblot on PopC and CRC sera. In the interim, sera were stored at $80{ }^{\circ} \mathrm{C}$. An effort was made to keep thaw-refreeze effects to a minimum.

Assays were interpreted using cutoffs pre-defined by the manufacturer. A test was deemed positive when either the IgM component, the IgG component, or both were positive. A test was considered negative when neither component was reactive. A test was considered equivocal when both components were equivocal, or when one component was equivocal and the other negative.

\section{Algorithms}

Three serodiagnostic algorithms were evaluated:

1. Single-tier: one of the EIAs/CLIA as sole test. Equivocal test results were classified as negative.

2. STTT: one of the EIAs/CLIA followed by immunoblot. This algorithm was evaluated in its standard form, i.e. equivocal test results in the EIAs were classified as positive, but equivocal test results in the immunoblot were classified as negative. 


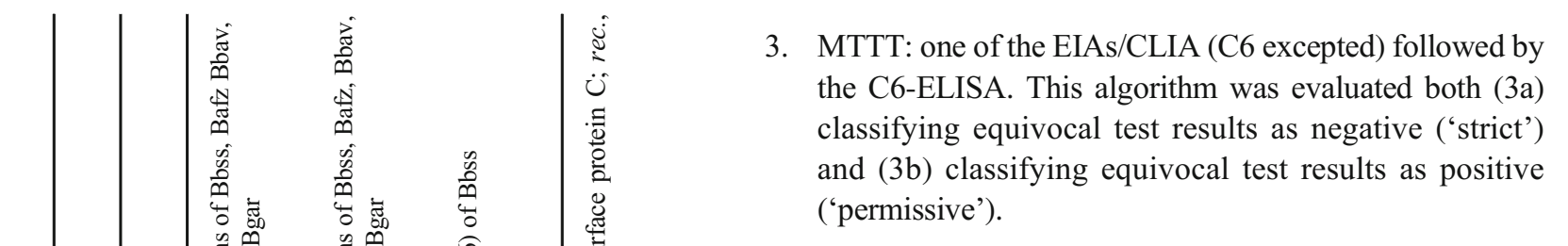

\section{Statistical analysis}

Diagnostic parameters of tests within each serum group were compared with one another using the McNemar test, or the exact McNemar where applicable. The 95\% confidence intervals of proportions (i.e. of the sensitivity and specificity) were determined using Clopper-Pearson. The differences in sensitivity and specificity of the permissive vs. strict variant of MTTT were assessed using a one-sample $t$ test, as algorithm interpretation rules prevented the McNemar test from being used in these comparisons. The same applied to the comparison of the MTTT-strict algorithm vs. the C6-ELISA as singletier test, and the comparison of MTTT-permissive vs. the MTTT-permissive IgG only. We performed subanalyses to assess consistency of reactivity across the various conditions in CRC sera. For all analyses, $p$ values $<0.05$ were considered statistically significant.

\section{Ethical statement}

The study was conducted according to the principles of the Declaration of Helsinki and in conformity with institutional regulations and guidelines. The study utilized only patient materials left over from standard clinical practice. Therefore, the Dutch Medical Research Involving Human Subjects Act does not apply to this study and no informed consent was asked from participants.

\section{Results}

Using the single-tier algorithm, three assays performed comparably with regard to sensitivity (78-81\%), except for the Liaison which showed a significantly lower sensitivity $(70 \%)$ than all other assays $(p<0.01)$ (Table 2). All singletier assays performed comparably with respect to specificity, both in PopC (89-94\%) and CRC sera (62-78\%) (Table 3). False positivity in CRC sera was mainly due to IgM crossreactivity in patients with acute $\mathrm{EBV}$ or CMV infection (data not shown).

Sensitivities of STTT protocols were much lower (4447\%). Specificities increased to 97\% for PopC sera and 88$92 \%$ for CRC sera. No significant differences were seen regarding sensitivity and specificity between the various assays as used in STTT (Tables 2 and 3). 
Table 2 Sensitivity of all algorithms

\begin{tabular}{|c|c|c|c|c|c|c|c|}
\hline \multicolumn{2}{|l|}{ Single-tier } & \multicolumn{2}{|l|}{ STTT } & \multicolumn{2}{|l|}{ MTTT-strict } & \multicolumn{2}{|c|}{ MTTT-permissive } \\
\hline $\begin{array}{l}\text { No. of true } \\
\text { positive } \\
\text { (sensitivity \%) }\end{array}$ & $95 \%-\mathrm{CI}$ & $\begin{array}{l}\text { No. of true } \\
\text { positive } \\
\text { (sensitivity \%) }\end{array}$ & $95 \%-\mathrm{CI}$ & $\begin{array}{l}\text { No. of true } \\
\text { positive } \\
\text { (sensitivity \%) }\end{array}$ & $95 \%-\mathrm{CI}$ & $\begin{array}{l}\text { No. of true } \\
\text { positive } \\
\text { (sensitivity \%) }\end{array}$ & $95 \%-\mathrm{CI}$ \\
\hline
\end{tabular}

EM sera $(n=228)$

Enz1 $180(78.9)$

Enz2 $184(80.7)^{1}$

73.1-84.1 Enz1/RecomL 106 (46.5)

75.0-85.6 Enz2/RecomL 106 (46.5)

Lia $160(70.2)^{1}$

63.8-76.0 Lia/RecomL

$101(44.3)$

C6 $178(78.1)^{*}$

72.1-83.3 C6/RecomL

$102(44.7)$
39.9-53.2 Enz1/C6 $162(71.1)^{1,2}$

39.9-53.2 Enz2/C6 $166(72.8)^{1,2}$

$64.7-76.8173(75.9)^{1,4,5}$

$66.5-78.5177(77.6)^{1,4,5}$

$69.8-81.3$

37.7-51.0 Lia/C6 $153(67.1)^{1,2}$

$60.6-73.2166(72.8)^{1,3,4,5}$

$71.7-82.9$

$38.2-51.4$

${ }^{1} p<0.01$ as compared with equivalent STTT using (McNemar/exact McNemar) $\mid\left({ }^{*} \mathrm{C} 6\right.$ vs. C6/RecomL $\left.p=0.016\right)$

${ }^{2} p<0.01$ as compared with single-tier C6-ELISA (one-sample $t$ test)

${ }^{3} p<0.01$ as compared with single-tier C6 (exact McNemar)

${ }^{4} p<0.01$ as compared with equivalent MTTT-strict (one-sample $t$ test)

${ }^{5}$ Not significant as compared with equivalent single-tier (exact McNemar)

A test result was considered to be (true) positive when either the IgM component, IgG component or both were positive

95\%-CI, 95\% confidence interval; STTT, standard two-tier testing; MTTT, modified two-tier testing; Enz1, Enzygnost-1; Enz2, Enzygnost-2; Lia, Liaison; C6, C6-ELISA; RecomL, RecomLine; EM, erythema migrans; PopC, population control; $C R C$, cross-reactive control; strict, counting equivocal EIA results as negative; permissive, counting equivocal EIA results as positive

We used MTTT in two variants: classifying equivocal results as negative ("strict") and classifying equivocal results as positive ("permissive"). The highest sensitivity within the
MTTT algorithm was achieved using the permissive variant with the Enzygnost-2 in the first tier, and the C6-ELISA in the second (77.6\%). The highest specificity was achieved using

Table 3 Specificity of all algorithms

\begin{tabular}{|c|c|c|c|c|c|c|c|}
\hline \multicolumn{2}{|l|}{ Single-tier } & \multicolumn{2}{|l|}{ STTT } & \multicolumn{2}{|l|}{ MTTT-strict } & \multicolumn{2}{|c|}{ MTTT-permissive } \\
\hline $\begin{array}{l}\text { No. of true } \\
\text { negative } \\
\text { (specificity \%) }\end{array}$ & $95 \%-\mathrm{CI}$ & $\begin{array}{l}\text { No. of true } \\
\text { negative } \\
\text { (specificity \%) }\end{array}$ & $95 \%-\mathrm{CI}$ & $\begin{array}{l}\text { No. of true } \\
\text { negative } \\
\text { (specificity \%) }\end{array}$ & $95 \%-C I$ & $\begin{array}{l}\text { No. of true } \\
\text { negative } \\
\text { (specificity \%) }\end{array}$ & $95 \%-\mathrm{CI}$ \\
\hline
\end{tabular}

PopC sera $(n=231)$

\begin{tabular}{|c|c|c|c|c|c|c|c|c|c|c|}
\hline Enz1 & $206(89.2)^{1}$ & $84.4-92.9$ & Enz1/RecomL & $224(97.0)$ & $93.9-98.8$ & Enz1/C6 & $223(96.5)^{2}$ & $93.3-98.5$ & $221(95.7)^{4,5}$ & $92.2-97.9$ \\
\hline Enz2 & $206(89.2)^{1}$ & $84.4-92.9$ & Enz2/RecomL & $224(97.0)$ & $93.9-98.8$ & Enz2/C6 & $223(96.5)^{2}$ & $93.3-98.5$ & $222(96.1)^{3,4,5}$ & $92.7-98.2$ \\
\hline Lia & $210(90.9)^{1}$ & $86.4-94.3$ & Lia/RecomL & $225(97.4)$ & $94.4-99.0$ & $\mathrm{Lia} / \mathrm{C6}$ & $223(96.5)^{2}$ & $93.3-98.5$ & $220(95.2)^{*, 5}$ & $91.6-97.6$ \\
\hline C6 & $216(93.5)^{1}$ & $89.5-96.3$ & C6/RecomL & $225(97.4)$ & $94.4-99.0$ & & & & & \\
\hline \multicolumn{11}{|c|}{ CRC sera $(n=50)$} \\
\hline Enz1 & $33(66.0)^{1}$ & $51.2-78.8$ & Enz1/RecomL & $45(90.0)$ & $78.2-96.7$ & Enz1/C6 & $43(86.0)^{2}$ & $73.3-94.2$ & $41(82.0)^{4,5}$ & $68.6-91.4$ \\
\hline Enz2 & $33(66.0)^{1}$ & $51.2-78.8$ & Enz2/RecomL & $45(90.0)$ & $78.2-96.7$ & Enz2/C6 & $43(86.0)^{2}$ & $73.3-94.2$ & $41(82.0)^{4,5}$ & $68.6-91.4$ \\
\hline Lia & $31(62.0)^{1}$ & $47.2-75.4$ & Lia/RecomL & $44(88.0)$ & $75.7-95.5$ & $\mathrm{Lia} / \mathrm{C6}$ & $43(86.0)^{2}$ & $73.3-94.2$ & $42(84.0)^{4,5}$ & $70.9-92.8$ \\
\hline C6 & $39(78.0)^{1}$ & $64.0-88.5$ & C6/RecomL & $46(92.0)$ & $80.0-97.8$ & & & & & \\
\hline
\end{tabular}

${ }^{1} p<0.01$ as compared with equivalent STTT (McNemar/exact McNemar)

${ }^{2} p<0.05$ as compared with single-tier C6-ELISA (one-sample $t$ test)

${ }^{3} p<0.05$ as compared with single-tier C6 (exact McNemar)

${ }^{4} p<0.01$ as compared with equivalent single-tier (exact McNemar) $\mid$ (*Lia vs. Lia/C6 (permissive) $p=0.021$ )

${ }^{5}$ Not significant as compared with equivalent MTTT-strict (one-sample $t$ test)

A test result was considered to be (true) negative when both the $\operatorname{IgM}$ and the $\operatorname{IgG}$ component were negative. A test result was considered to be (false) positive when either or both components were positive

95\%-CI, 95\% confidence interval; STTT, standard two-tier testing; MTTT, modified two-tier testing; Enz1, Enzygnost-1; Enz2, Enzygnost-2; Lia, Liaison; C6, C6-ELISA; RecomL, RecomLine; $E M$, erythema migrans; $P o p C$, population control; $C R C$, cross-reactive control; strict, counting equivocal EIA results as negative; permissive, counting equivocal EIA results as positive 
Table 4 Diagnostic odds ratios of all algorithms

\begin{tabular}{|c|c|c|c|c|c|c|}
\hline & $\begin{array}{l}\text { Single-tier } \\
\text { DOR }(95 \% \text {-CI })\end{array}$ & & $\begin{array}{l}\text { STTT } \\
\text { DOR }(95 \%-C I)\end{array}$ & & $\begin{array}{l}\text { MTTT-strict } \\
\text { DOR }(95 \% \text {-CI) }\end{array}$ & $\begin{array}{l}\text { MTTT- } \\
\text { permissive } \\
\text { DOR }(95 \%-C I)\end{array}$ \\
\hline \multicolumn{7}{|c|}{ DOR with PopC sera } \\
\hline Enz1 & $30.9(18.1-52.1)$ & Enz1/RecomL & $27.8(12.5-61.6)$ & Enz1/C6 & $68.4(32.0-146.5)$ & $69.5(34.4-140.3)$ \\
\hline Enz2 & $34.5(20.3-58.4)$ & Enz2/RecomL & $27.8(12.5-61.6)$ & Enz2/C6 & $74.6(34.8-160.1)$ & $85.6(41.0-178.6)$ \\
\hline Lia & $23.5(13.8-40.0)$ & Lia/RecomL & $29.8(12.7-69.9)$ & $\mathrm{Lia} / \mathrm{C6}$ & $56.9(26.6-121.3)$ & $53.6(27.3-104.9)$ \\
\hline C6 & $51.3(27.9-94.4)$ & C6/RecomL & $30.3(12.9-71.2)$ & & & \\
\hline \multicolumn{7}{|c|}{ DOR with CRC sera } \\
\hline Enz1 & $7.3(3.7-14.2)$ & Enz1/RecomL & $7.8(2.9-20.4)$ & Enz1/C6 & $15.1(6.5-35.2)$ & $14.3(6.5-31.3)$ \\
\hline Enz2 & $8.1(4.2-15.9)$ & Enz2/RecomL & $7.8(2.9-20.4)$ & Enz2/C6 & $16.5(7.0-38.5)$ & $15.8(7.2-34.6)$ \\
\hline Lia & $3.8(2.0-7.3)$ & Lia/RecomL & $5.8(2.4-14.2)$ & $\mathrm{Lia} / \mathrm{C6}$ & $12.5(5.4-29.2)$ & $14.0(6.2-31.6)$ \\
\hline C6 & $21.6(6.0-26.4)$ & C6/RecomL & $9.3(3.2-26.7)$ & & & \\
\hline
\end{tabular}

DOR, diagnostic odds ratio; 95\%-CI, 95\% confidence interval; STTT, standard two-tier testing; MTTT, modified two-tier testing; Enzl, Enzygnost-1; Enz2, Enzygnost-2; Lia, Liaison; C6, C6-ELISA; RecomL, RecomLine; EM, erythema migrans; PopC, population control; CRC, cross-reactive control; strict, counting equivocal EIA results as negative; permissive, counting equivocal EIA results as positive

the strict variant of the MTTT algorithm, irrespective of the first-tier EIA that was used $(96.5 \%)$. In both variants, the sensitivity of the combination Enzygnost-2/C6 was better than that of the combination Liaison/C6 (strict $p=0.003$; permissive $p=0.007)$. Other comparisons within each algorithm/ variant yielded no significant differences (Tables 2 and 3).

When comparing the single-tier, MTTT-strict, or MTTTpermissive algorithms to STTT, we found sensitivity of all these algorithms to be significantly better than the equivalent STTT algorithm. The specificity of all assays in the single-tier algorithm was significantly lower than that of STTT. However, the specificity of both MTTT strategies in the population controls and in controls with a potentially crossreactive condition was comparable with that of STTT.

Comparing the permissive variant of the MTTT algorithm to the strict variant, we found that the permissive strategy resulted in a higher sensitivity for all assays $(p<0.01)$. This increase in sensitivity was $4.8 \%$ (95\% CI 2.0-7.6) for the Enzygnost-1/C6 and Enzygnost-2/C6, and 5.7\% (95\% CI 2.6-8.7) for the Liaison/C6. Interestingly, specificity in both population controls and controls with a potentially crossreactive illness was comparable between the two variants. The MTTT-permissive algorithm also outperformed equivalent single-tier assays, as all MTTT-permissive combinations had comparable sensitivity, but a higher specificity than their single-tier equivalent.

Comparing all MTTT combinations to the "benchmark" C6-ELISA, we found unsurprisingly that the assays in the strict variant had a significantly better specificity than the single-tier C6-ELISA. These combinations did all have significantly lower sensitivity. As previously mentioned, all MTTTpermissive variants had a better sensitivity than their equivalent strict variant, and two of such combinations (Enz1/C6 and
Enz2/C6) even had comparable sensitivity with the single-tier C6-ELISA. Importantly, for the best scoring combination (Enz2/C6), this did not come at the cost of a loss of specificity, which was still better than that of the single-tier C6-ELISA (specificity PopC C6 93.5\% vs. C6/Enz2 96.1\%, $p=0.03$ ). All comparisons between MTTT variants and the C6-ELISA are given in Tables 2 and 3 .

The diagnostics odds ratios for the various assays and algorithms are given in Table 4.

Among the screening EIAs, the relative contribution of the IgM component was highest in the Enzygnost-1 assay. That assay's IgG component, which does not contain VlsE, performed rather poorly (Fig. 2a, orange and pink bars combined), as did the IgM component of the Liaison, which relies on recombinant antigens only (Fig. 2a, orange and green bars combined). Use of MTTT was most useful for correct classification of IgM-positive PopC sera (Fig. 2b) and also filtered out a significant number of IgM false-positive CRC sera (Fig. 2c). Based on Fig. $2 \mathrm{a}-\mathrm{c}$, it could be hypothesized that using only the IgG component of each first-tier assay would result in a large improvement of specificity in CRC sera with only a minor decrease in sensitivity. Diagnostic parameters of this MTTT-permissive IgG-only variant are given in Table 5. This variant without a separate $\operatorname{IgM}$ component had the expected increase in specificity in CRC sera, but this did come at the cost of a statistically significant decrease in sensitivity for all combinations. Within the IgGonly variant, the Enz2-IgG/C6 outperformed both other combinations (both comparisons $p<0.01$ ). Further subanalysis of both MTTT algorithms showed that Enzygnost-2-IgG outperformed Liaison-IgG in terms of sensitivity $(p=0.041)$, while having comparable 

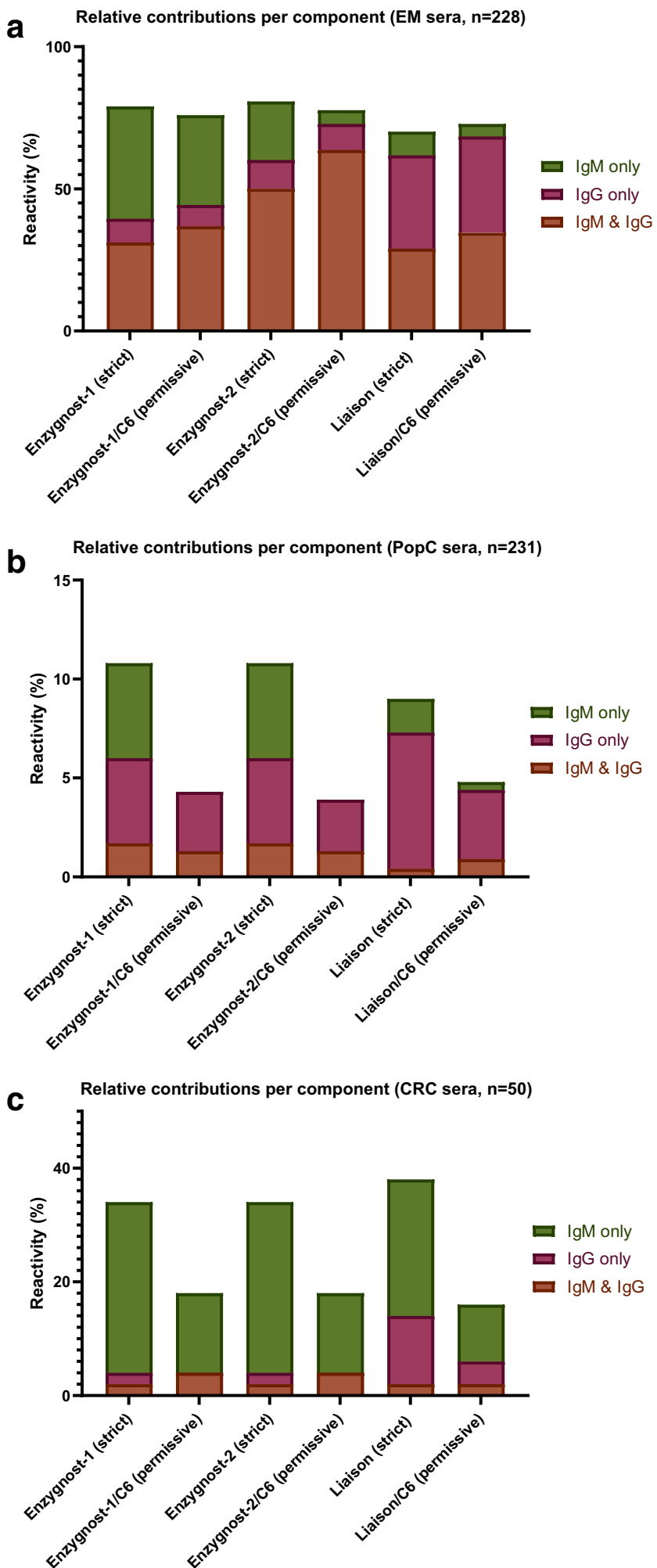

Fig. 2 Reactivity per component - single-tier \& MTTT-permissive (C6 and RecomL not shown) a EM sera, percentages calculated from $n=228$. b PopC sera, percentages calculated from $n=231$. $\mathbf{c}$ CRC sera, percentages calculated from $n=50$ specificity in PopC and CRC sera, but only in the permissive variant (strict variant, sensitivity Enz1-IgG vs. Lia$\operatorname{IgG} p=0.664$ ) (Supplementary Table 1).

Subanalysis of IgG-blot positive vs. IgG-blot negative EM sera showed that the additional value of VlsE in the IgG-EIAs was restricted to blot-negative sera, i.e. early infection (IgGblot NEG, Enz2-IgG/Lia-IgG vs. Enz1-IgG $p<0.01$; IgG-blot POS, all comparisons $p>0.05$ ) (Supplementary Table 2).

\section{Discussion}

In our study, we have evaluated various algorithms of singletier testing or MTTT compared with STTT in European patients with early localized LB. Previous studies conducted in the USA have shown that MTTT protocols generally improve sensitivity of serological tests for LB without losing specificity $[7,13-15]$. Our results show that the same holds true for the European situation, even when the screening EIA contains VlsE and the confirmatory test is the C6-ELISA, which functions with the IR6 peptide of VlsE.

Overall, the best diagnostic parameters were found using the Enzygnost-2 in the first tier, even though differences with the other assays within any algorithm were mostly non-significant. The highest sensitivity was found when using this assay as a standalone single-tier test. As was to be expected, this did result in a markedly lower specificity. Conversely, STTT had the best specificity, but lacked sensitivity in the EM sera that were used. The sensitivity of STTT in our study was comparable with the reported sensitivity of serological testing in EM patients in a meta-analysis by Leeflang and colleagues [11], and we therefore consider it to be representative of EM patients in general.

The algorithms of MTTT that we studied all showed marked improvement of sensitivity as compared with STTT, without significant loss of specificity. Because MTTT is quite new in Europe, we also investigated which "rules" should be used for assay interpretation. Interestingly, we found that classifying equivocal results as positive resulted in an increase of sensitivity and only a negligible and non-significant decrease in specificity. For each assay, this permissive approach maintained comparable sensitivity with their single-tier equivalents, but with a significantly better specificity.

Finally, it must be noted that the best performing combination (Enzynost-2/C6 in the permissive variant) had a comparable sensitivity with the "benchmark" C6-ELISA, but a significantly better specificity in population controls. While the absolute differences were small (C6 93.5\% vs. Enz2/C6 $96.1 \%$ ), this is still of great significance in the field of LB diagnostics. Borrelia serology is frequently requested for patients with a low pre-test probability of having LB; hence, the impact of a small improvement in specificity on the eventual positive predictive value can be substantial. This implies that 
Table 5 Diagnostic parameters of algorithms with IgG component only
EM sera $(n=228)$

MTTT-permissive

\begin{tabular}{|c|c|c|c|c|c|}
\hline & $\begin{array}{r}\text { No. of true positive } \\
\text { (sensitivity \%) }\end{array}$ & $95 \%$-CI & & $\begin{array}{r}\text { No. of true positive } \\
\text { (sensitivity \%) }\end{array}$ & $95 \%-\mathrm{CI}$ \\
\hline Enz1/C6 & $173(75.9)$ & $69.8-81.3$ & Enz1-IgG/C6 & $101(44.3)^{*}$ & $37.7-51.0$ \\
\hline Enz2/C6 & $177(77.6)$ & $71.7-82.9$ & Enz2-IgG/C6 & $166(72.8)^{*}$ & $66.5-78.5$ \\
\hline Lia/C6 & $166(72.8)$ & $66.5-78.5$ & Lia-IgG/C6 & $156(68.4)^{*}$ & $62.0-74.4$ \\
\hline \multicolumn{6}{|c|}{ PopC sera $(n=231)$} \\
\hline \multicolumn{3}{|c|}{ MTTT-permissive } & & \multicolumn{2}{|c|}{ MTTT-permissive IgG-only } \\
\hline & $\begin{array}{r}\text { No. of true negative } \\
\text { (specificity \%) }\end{array}$ & $95 \%$-CI & & $\begin{array}{r}\text { No. of true negative } \\
\text { (specificity \%) }\end{array}$ & $95 \%-\mathrm{CI}$ \\
\hline Enz1/C6 & $221(95.7)$ & $92.2-97.9$ & Enz1-IgG/C6 & $221(95.7)$ & $92.2-97.9$ \\
\hline Enz2/C6 & $222(96.1)$ & $92.7-98.2$ & Enz2-IgG/C6 & $222(96.1)$ & $92.7-98.2$ \\
\hline Lia/C6 & $220(95.2)$ & $91.6-97.6$ & Lia-IgG/C6 & $221(95.7)$ & $92.2-97.9$ \\
\hline \multicolumn{6}{|c|}{ CRC sera $(n=50)$} \\
\hline \multicolumn{2}{|c|}{ MTTT-permissive } & & & \multicolumn{2}{|c|}{ MTTT-permissive IgG-only } \\
\hline & $\begin{array}{r}\text { No. of true negative } \\
\text { (specificity \%) }\end{array}$ & $95 \%$-CI & & $\begin{array}{r}\text { No. of true negative } \\
\text { (specificity \%) }\end{array}$ & $95 \%$-CI \\
\hline Enz1/C6 & $41(82.0)$ & $68.6-91.4$ & Enz1-IgG/C6 & $48(96.0)^{*}$ & $86.3-99.5$ \\
\hline Enz2/C6 & $41(82.0)$ & $68.6-91.4$ & Enz2-IgG/C6 & $48(96.0)^{*}$ & $86.3-99.5$ \\
\hline $\mathrm{Lia} / \mathrm{C6}$ & $42(84.0)$ & $70.9-92.8$ & Lia-IgG/C6 & $47(94.0)^{*}$ & $83.5-98.8$ \\
\hline
\end{tabular}

$* p<0.05$ as compared with equivalent MTTT-permissive (one-sample $t$ test)

95\%-CI, 95\% confidence interval; MTTT, modified two-tier testing; Enz1, Enzygnost-1; Enz2, Enzygnost-2; Lia, Liaison; C6, C6-ELISA; EM, erythema migrans; PopC, population control; $C R C$, cross-reactive control; permissive, counting equivocal EIA results as positive; $\operatorname{Ig} G$ only, using only the $\operatorname{IgG}$ component of the first-tier assay two-tier testing is still advantageous, in spite of the C6ELISA's excellent diagnostic parameters. It must be noted that further improvements to the diagnostic parameters of multitier Borrelia serology may be possible when using more innovative ways of combining assays or assays' components than the simple Boolean logic (i.e. AND/OR) we have employed in the current study $[19,20]$. Our findings do not support dropping the IgM component from the first-tier assays, even in assays which have VlsE in their IgG component, even though such a move has recently been gaining popularity [21, 22].

One might argue that serological testing for an EM is not good clinical practice. After all, clinical guidelines state that an EM is considered a clinical diagnosis [1-3]. However, this is in part because current serological algorithms lack adequate sensitivity. Taking into account our findings, it may be prudent to further investigate the applicability of MTTT for patients with early LB. Of course, treatment of a cutaneous lesion which is recognized as an EM should not be delayed by serological confirmation, as antibodies may not always have formed. However, both our and previous studies show that requests for serology are nonetheless frequent in the setting of Dutch general practice, even in patients with clear-cut EM [8]. This implies that patients and clinicians value the added certainty that laboratory work-up gives to the physician's clinical assessment. Laboratory testing may be especially relevant in situations where the LB diagnosis is not clear-cut, for example, when skin lesions do not resemble a typical EM, or if patients present with only generalized symptoms without a skin lesion [23, 24]. Considering the improved diagnostic parameters of MTTT, serology may serve to aid in the diagnosis of these situations. All of the aforementioned does not negate the recommendation, however, that cutaneous lesions should be treated as an EM without awaiting serological outcomes, if the clinician recognizes them as such. Finally, it is important to note once more that the true value of any MTTT algorithm will depend on the setting in which the algorithm is used (i.e. the pre-test probability and resulting positive and negative predictive values).

Our study also indicates that the $\operatorname{IgM}$ and $\operatorname{IgG}$ components of the different assays rely on different antigens for their reactivity. The IgG assay without VlsE was substantially less reactive in EM sera, as was the IgM assay which did not contain a whole cell extract but relied on recombinant antigens only. This last finding is in agreement with a recent study from Northern Europe [25]. Our subanalysis allows for a tentative hypothesis that the VlsE from the Enzygnost-2, consisting of three species, performs better than Liaison's Bbav-VlsE; 
however, these results depended on the algorithm used (strict vs. permissive). Further studies should confirm whether addition of VlsE from different species improves diagnostic accuracy.

A limitation of our study is that we did not include a polymerase chain reaction (PCR) or culture on skin biopsies obtained from the EM to verify the presence of Bbsl in these lesions. However, the sensitivity of culture or PCR is not perfect $[26,27]$. Excluding EMs in which the spirochete cannot be directly detected may therefore lead to an overestimation of the sensitivity. Branda and colleagues [13] also found a trend towards higher sensitivity of their MTTT algorithms in patients with culture-confirmed EM compared with culturenegative EMs. Our study might also have benefitted from multiple sera from the same patient from both the acute and convalescent phase; however, these were not available for a sufficient number of patients in this convenience sample. In contrast, a strength of our study was that the population controls were drawn from a representative sample of the population as a whole, whereas many studies use blood bank donors as controls. Blood bank donors are inherently a healthier subset of the entire population, with the potential for bias towards a different background seroprevalence.

Due to the rarity of definite and probable disseminated LB, we could not collect sufficient sera during the study period to include these in the study as well. Even though one of the major advantages of MTTT (i.e. improved sensitivity) primarily applies to early (localized) LB, it is imperative that future studies compare different MTTT algorithms for patients with disseminated LB as well. Even though the expected improvement in diagnostic parameters will likely be smaller, other advantages of MTTT will still apply: EIAs require less labor and hence lower costs of LB serology, and due to their quantitative nature have a decreased risk of inter-assay variation [28].

As a final remark, it is evident that MTTT protocols are not the solution to some of the other inherent problems of serology for LB diagnostics. MTTT does not solve the problem of distinguishing between an active and past infection after IgGseroconversion, and all serology still depends on indirect methods of detection, as opposed to direct detection of the causative microorganism. MTTT protocols may, however, be a partial solution to some of the problems surrounding serology, which cause controversy among doctors and patients.

Acknowledgments The authors thank Prof. PMM (Patrick) Bossuyt, $\mathrm{PhD}$; MMG (Mariska) Leeflang, PhD; Prof. AH (Koos) Zwinderman, $\mathrm{PhD}$; and Carolien Duetz, MD, for their epidemiological and statistical contributions to this manuscript.

Funding information The Liaison, Enzygnost, and RecomLine assays were supplied free of charge by their manufacturers. This study was performed without any other external funding. None of the test manufacturers had any role in the design or execution of the study, the analysis and interpretation of data, or the decision to publish. JWH's work on an unrelated project was supported by the European Union's regional development fund (INTERREG) as part of the NorthTick project.

Data availability The dataset generated during and/or analyzed during the current study is available from the corresponding author on reasonable request.

\section{Compliance with ethical standards}

Conflict of interest MEB and JWH report that they collaborate with Bio-Rad Laboratories to investigate a novel serological assay for LB which was not included in this study. MEB and JWH are also involved in an unrelated study investigating the diagnostic parameters of several cellular assays for LB. Neither has received any personal financial compensation from any of said companies for these projects. The other authors report no potential conflicts of interest.

Ethical approval The Dutch Medical Research Involving Human Subjects Act does not apply to this study, as it was performed using deidentified data and leftover materials from routine clinical procedures. This study was performed in conformity with the Declaration of Helsinki and institutional regulations and guidelines.

Consent to participate As the Dutch Medical Research Involving Human Subjects Act does not apply to this study, no informed consent was required.

\section{Consent to publish Not applicable.}

Open Access This article is licensed under a Creative Commons Attribution 4.0 International License, which permits use, sharing, adaptation, distribution and reproduction in any medium or format, as long as you give appropriate credit to the original author(s) and the source, provide a link to the Creative Commons licence, and indicate if changes were made. The images or other third party material in this article are included in the article's Creative Commons licence, unless indicated otherwise in a credit line to the material. If material is not included in the article's Creative Commons licence and your intended use is not permitted by statutory regulation or exceeds the permitted use, you will need to obtain permission directly from the copyright holder. To view a copy of this licence, visit http://creativecommons.org/licenses/by/4.0/.

\section{References}

1. Wormser GP, Dattwyler RJ, Shapiro ED, Halperin JJ, Steere AC, Klempner MS et al (2006) The clinical assessment, treatment, and prevention of Lyme disease, human granulocytic anaplasmosis, and babesiosis: clinical practice guidelines by the Infectious Diseases Society of America. Clin Infect Dis 43(9):1089-1134

2. Dessau RB, van Dam AP, Fingerle V, Gray J, Hovius JW, Hunfeld KP et al (2018) To test or not to test? Laboratory support for the diagnosis of Lyme borreliosis: a position paper of ESGBOR, the ESCMID study group for Lyme borreliosis. Clin Microbiol Infect 24(2):118-124

3. Eldin C, Raffetin A, Bouiller K, et al. Review of European and American guidelines for the diagnosis of Lyme borreliosis. Med Mal Infect.2019;49(2):121-132. https://doi.org/10.1016/j.medmal. 2018.11.011 
4. Branda JA, Aguero-Rosenfeld ME, Ferraro MJ, Johnson BJ, Wormser GP, Steere AC (2010) 2-tiered antibody testing for early and late Lyme disease using only an immunoglobulin $\mathrm{G}$ blot with the addition of a VlsE band as the second-tier test. Clin Infect Dis 50(1):20-26

5. Magnarelli LA, Lawrenz M, Norris SJ, Fikrig E (2002) Comparative reactivity of human sera to recombinant VlsE and other Borrelia burgdorferi antigens in class-specific enzyme-linked immunosorbent assays for Lyme borreliosis. J Med Microbiol 51(8):649-655

6. Liang FT, Aberer E, Cinco M, Gern L, Hu CM, Lobet YN et al (2000) Antigenic conservation of an immunodominant invariable region of the VlsE lipoprotein among European pathogenic genospecies of Borrelia burgdorferi SL. J Infect Dis 182(5):14551462

7. Branda JA, Linskey K, Kim YA, Steere AC, Ferraro MJ (2011) Two-tiered antibody testing for Lyme disease with use of 2 enzyme immunoassays, a whole-cell sonicate enzyme immunoassay followed by a VlsE C6 peptide enzyme immunoassay. Clin Infect Dis 53(6):541-547

8. Coumou J, Hovius JW, van Dam AP (2014) Borrelia burgdorferi sensu lato serology in the Netherlands: guidelines versus daily practice. Eur J Clin Microbiol Infect Dis 33(10):1803-1808

9. Steere AC, Strle F, Wormser GP, Hu LT, Branda JA, Hovius JW et al (2016) Lyme borreliosis. Nat Rev Dis Primers 2:16090

10. Goettner G, Schulte-Spechtel U, Hillermann R, Liegl G, Wilske B, Fingerle V (2005) Improvement of Lyme borreliosis serodiagnosis by a newly developed recombinant immunoglobulin $\mathrm{G}(\mathrm{IgG})$ and IgM line immunoblot assay and addition of VlsE and DbpA homologues. J Clin Microbiol 43(8):3602-3609

11. Leeflang MMG, Ang CW, Berkhout J, Bijlmer HA, Van Bortel W, Brandenburg AH et al (2016) The diagnostic accuracy of serological tests for Lyme borreliosis in Europe: a systematic review and meta-analysis. BMC Infect Dis 16:140

12. Wormser GP, Schriefer M, Aguero-Rosenfeld ME, Levin A, Steere AC, Nadelman RB et al (2013) Single-tier testing with the C6 peptide ELISA kit compared with two-tier testing for Lyme disease. Diagn Microbiol Infect Dis 75(1):9-15

13. Branda JA, Strle K, Nigrovic LE, Lantos PM, Lepore TJ, Damle NS et al (2017) Evaluation of modified 2-tiered serodiagnostic testing algorithms for early Lyme disease. Clin Infect Dis 64(8):10741080

14. Pegalajar-Jurado A, Schriefer ME, Welch RJ, et al. Evaluation of Modified Two-Tiered Testing Algorithms for Lyme Disease Laboratory Diagnosis Using Well-Characterized Serum Samples. J Clin Microbiol. 2018;56(8):e01943-17. Published 2018 Jul 26. https://doi.org/10.1128/JCM.01943-17

15. Molins CR, Delorey MJ, Sexton C, Schriefer ME (2016) Lyme Borreliosis serology: performance of several commonly used laboratory diagnostic tests and a large resource panel of wellcharacterized patient samples. J Clin Microbiol 54(11):2726-2734

16. (2019) FDA clears new indications for existing Lyme disease tests that may help streamline diagnoses [press release]. Food and Drug Administration
17. (2013) CBO-richtlijn Lymeziekte: National Institute of Public Health and the Environment; [Dutch guideline for Lyme disease, accessed on 14-04-2020]. Available from: https://www.rivm.nl/ documenten/cbo-richtlijn-lymeziekte-juli-2013

18. van der Klis FR, Mollema L, Berbers GA, de Melker HE, Coutinho RA (2009) Second national serum bank for population-based seroprevalence studies in the Netherlands. Neth J Med 67(7):301-308

19. Pepe MS. The Statistical Evaluation of Medical Tests for Classification and Prediction. Oxford: OUP Oxford; 2003. Chapter 9.3: Combining multiple test results; p.267-76

20. Dessau RB, Ejlertsen T, Hilden J (2010) Simultaneous use of serum IgG and IgM for risk scoring of suspected early Lyme borreliosis: graphical and bivariate analyses. APMIS. 118(4):313-323

21. Dessau RB (2013) Diagnostic accuracy and comparison of two assays for Borrelia-specific IgG and IgM antibodies: proposals for statistical evaluation methods, cut-off values and standardization. J Med Microbiol 62(Pt 12):1835-1844

22. Hillerdal H, Henningsson AJ. Serodiagnosis of Lyme borreliosis is $\operatorname{IgM}$ in serum more harmful than helpful? Presented at: International Symposium on Tick-Borne Pathogens and Disease; 2019 Sept 8-11; Vienna, Austria.

23. Strle F, Nadelman RB, Cimperman J, Nowakowski J, Picken RN, Schwartz I et al (1999) Comparison of culture-confirmed erythema migrans caused by Borrelia burgdorferi sensu stricto in New York State and by Borrelia afzelii in Slovenia. Ann Intern Med 130(1): 32-36

24. Strle F, Ruzic-Sabljic E, Logar M, Maraspin V, Lotric-Furlan S, Cimperman J et al (2011) Comparison of erythema migrans caused by Borrelia burgdorferi and Borrelia garinii. Vector Borne Zoonotic Dis 11(9): $1253-1258$

25. Lager M, Dessau RB, Wilhelmsson P, Nyman D, Jensen GF, Matussek A et al (2019) Serological diagnostics of Lyme borreliosis: comparison of assays in twelve clinical laboratories in Northern Europe. Eur J Clin Microbiol Infect Dis 38(10):19331945

26. Liveris D, Schwartz I, McKenna D, Nowakowski J, Nadelman R, Demarco J et al (2012) Comparison of five diagnostic modalities for direct detection of Borrelia burgdorferi in patients with early Lyme disease. Diagn Microbiol Infect Dis 73(3):243-245

27. Cerar T, Ruzic-Sabljic E, Glinsek U, Zore A, Strle F (2008) Comparison of PCR methods and culture for the detection of Borrelia spp. in patients with erythema migrans. Clin Microbiol Infect 14(7):653-658

28. Wormser GP, Levin A, Soman S, Adenikinju O, Longo MV, Branda JA (2013) Comparative cost-effectiveness of two-tiered testing strategies for serodiagnosis of Lyme disease with noncutaneous manifestations. J Clin Microbiol 51(12):4045-4049

Publisher's note Springer Nature remains neutral with regard to jurisdictional claims in published maps and institutional affiliations. 